\title{
Age paternel et descendance
}

\author{
M. AUROUX \\ Andrologie et Biologie de la Procréation CHU BICETRE \\ (Univ. PARIS-SUD) 94275 KREMLIN BICETRE
}

\section{RÉSUMÉ}

Le vieillissement testiculaire concerne à la fois l'individu et sa descendance. Chez l'individu, les modifications touchant la vascularisation, les cellules endocrines, la barrière hémato-testiculaire et les cellules de Sertoli entraînent une chute du nombre des spermatozoïdes ainsi qu'une altération de leur morphologie et de leur mobilité. Ces changements entraînent une diminution progressive de la fertilité. Chez la descendance, le vieillissement paternel augmente le risque de mutations autosomiques dominantes à l'origine de différentes malformations ou perturbations fonctionnelles et celui de certaines mutations récessives liées au sexe. Il semble en outre responsable d'une diminution de la longévité des filles. Enfin, chez l'animal et l'homme, le très jeune âge et le vieillissement du père s'accompagnent d'une diminution des fonctions cognitives de la progéniture. Lâge maternel ne paraît pas jouer de rôle dans ce phénomène. Dans l'ensemble, ces résultats posent le problème de l'âge optimum de la paternité.

Mots clés : Age paternel, spermatozö̈de, fertilité, mutations, progéniture

\section{INTRODUCTION}

Qu'il s'agisse de la jeunesse ou de la vieillesse, l'âge semble responsable, chez l'homme, d'une évolution particulière de la fertilité et d'un certain nombre de risques pour la descendance.

A une époque où, au moins dans nos pays occidentaux, la sexualité reproductrice commence de plus en plus tôt et se termine, pour les hommes, de plus en plus tard, ces données prennent un intérêt considérable. En outre, elles ne permettent plus, maintenant comme jadis, de faire de la femme l'unique responsable des difficultés de la conception ou des anomalies du conceptus dues à l'âge des gamètes.

\section{L'EVOLUTION DE LA FERTILITE}

Le vieillissement testiculaire se traduit par des modifications tissulaires, à la fois structurales et fonctionnelles. Un des témoins de ces modifications est l'appauvrissement progressif de la vascularisation, la densité des capillaires diminuant peu à peu à partir de la trentaine (figure 1) [1]. On sait aussi que les modifications des cellules endocrines aboutissent à une chute, d'ailleurs très variable, de la production des androgènes ; que des modifications enzymatiques aboutissent à une diminution d'efficacité de la barrière hémato-testiculaire et que le vieillissement des cellules de Sertoli aboutit à une chute de la production de Androgen-binding-protein [Revue gén. : 2]. L'ensemble de ces changements conduit à une lente diminution du nombre des spermatozoïdes produits, mise en évidence dès le XIXè siècle [3] et 
confirmée depuis (figure 2) [4]. Cependant on a montré que c'est moins le nombre des gamètes que leur qualité qui sous-tend la fertilité masculine. Or, de ce point de vue, les travaux de Schwartz et coll. [5] sont très révélateurs. Analysant la numération, la morphologie et la mobilité des spermatozoïdes chez des hommes de 20 à 59 ans, ces auteurs montrent que si la numération est relativement stable les paramètres qualitatifs, au contraire, évoluent en fonction de l'âge : les valeurs sont maximales entre 25 et 35 ans, croissantes avant, décroissantes après (figure 3 ). Le vieillissement, finalement, aboutit à une dégradation du nombre et de la qualité des spermatozoïdes, cette dernière étant touchée plus tôt que la quantité et n'étant pas satisfaisante, non plus, lorsque l'individu est très jeune, ce que l'on retrouve chez la souris [6] et le taureau [7].

Conséquence globale de cette évolution, la fertilité masculine diminue avec l'âge $[8,9]$, ce qui correspond aux enquêtes épidémiologiques concernant la fertilité féminine (figure 4) [9, 10]. Il reste maintenant à envisager les conséquences d'une telle situation pour la progéniture.

\section{AGE PATERNEL ET DESCENDANCE}

Qu'il soit masculin ou féminin, le vieillissement parental peut constituer un problème de reproduction pour les deux raisons suivantes:

- la fertilité diminue et le risque d'anomalies du conceptus augmente avec l'âge des parents;

- la contraception et les conditions socio-économiques actuelles permettent à certains couples d'attendre longtemps avant d'avoir leur premier enfant.

Pour sa part, le vieillissement paternel peut être responsable, dans les spermatozoïdes, d'anomalies chromosomiques de nombre ou de structure s'accompagnant parfois d'anomalies chez l'enfant. Il peut aussi être la cause de mutations dominantes ou récessives entraînant chez la descendance l'apparition de syndromes bien définis ou de modifications fonctionnelles parfois très subtiles, concernant par exemple certains mécanismes cérébraux.

\section{Anomalies chromosomiques}

Dans une population normale d'hommes âgés de 30 ans, environ $5 \%$ de spermatozoïdes présentent une non disjonction d'origine méiotique, ce qui conduit à une aneuploïdie $[11,12$, 13]. Pour certains auteurs, 5 à $10 \%$ des trisomies 21 seraient ainsi d'origine masculine [14, 15] mais, dans ce cas, le rôle de l'âge paternel est très discuté : perceptible pour les uns [16, $17,18]$, il est actuellement inappréciable pour les autres $[15,19,20]$. Environ $50 \%$ des Klinefelter seraient aussi, pour Hassold [21], dûs au vieillissement masculin. En revanche les autres aneuploïdies comme les trisomies 13 et 18, $47 \mathrm{XXX}$ et $47 \mathrm{XYY}$ ou la monosomie $45 \mathrm{X}$ ne seraient pas liées à l'âge paternel.

En ce qui concerne les anomalies de structure des chromosomes, on a montré qu'il existait une corrélation entre leur taux et l'augmentation de l'âge. Au dessus de 44 ans par exemple, il existe $13 \%$ de gamètes porteurs d'anomalies de structures. Cependant ces anomalies ne paraissent pas avoir d'effet sur la descendance [11]. Enfin, selon Hook et coll. [22], le vieillissement paternel s'accompagne d'une élévation du taux des translocations réciproques équilibrées chez le fœetus.

\section{Syndromes dominants et récessifs}

On sait depuis longtemps que le vieillissement paternel est associé à certaines mutations autosomiques dominantes se traduisant par des malformations macroscopiques comme l'achondroplasie [23], la maladie d'Apert, les syndromes de Marfan, de Crouzon, de Wardenburg, de Treacher-Collins, de Sotos, le syndrome oculo dento digital, l'acrodysostose, la progeria, les exostoses multiples, la dysplasie thanatophore (nanisme micromélique) et l'aniridie. Selon de nombreux auteurs, il est également impliqué dans la survenue d'anomalies plus discrètes comme la neurofibromatose de Recklinghausen (syndrome le plus fréquent : 1 pour 3000 naissances) [24] (Tableau 1) et dans celle de processus tumoraux comme la tumeur de Wilms, le rétinoblastome bilatéral, la polypose colique, la sclérose tubéreuse de Bourneville, la polykystose rénale et le naevus cellulaire, ou encore dans les perturbations métaboliques à l'origine de la rétinite pigmentaire, de l'ostéogénèse imparfaite (maladie des os de verre), de la fibrodysplasie ossifiante progressive, des syndromes de Costello ou de 


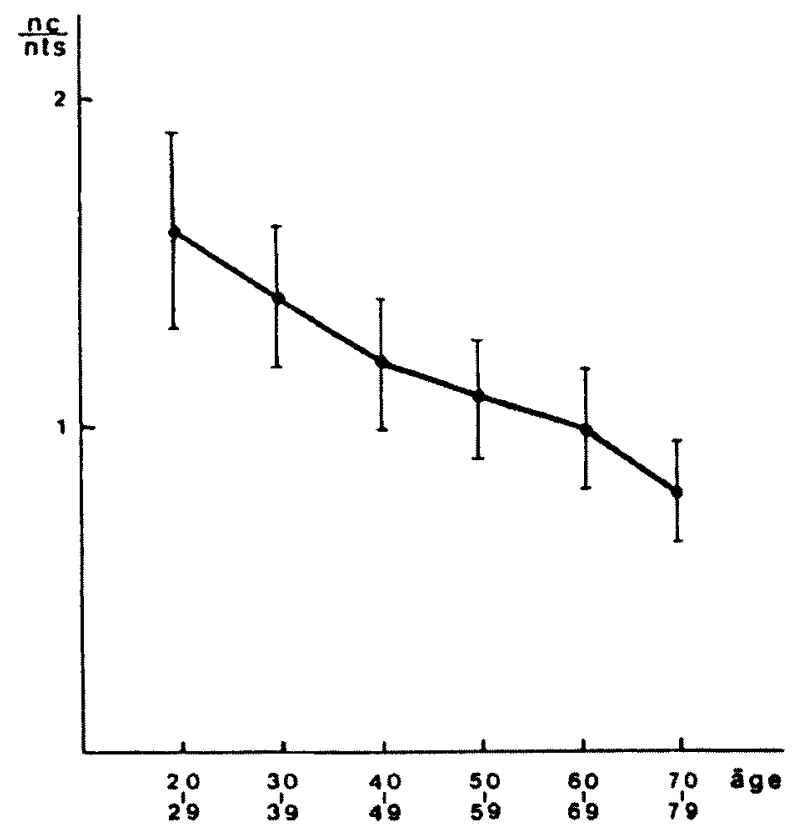

Figure 1 : Evolution du rapport "nombre de capillaires/nombre de tubes séminifères" en fonction de l'âge (années) chez l'homme [1].

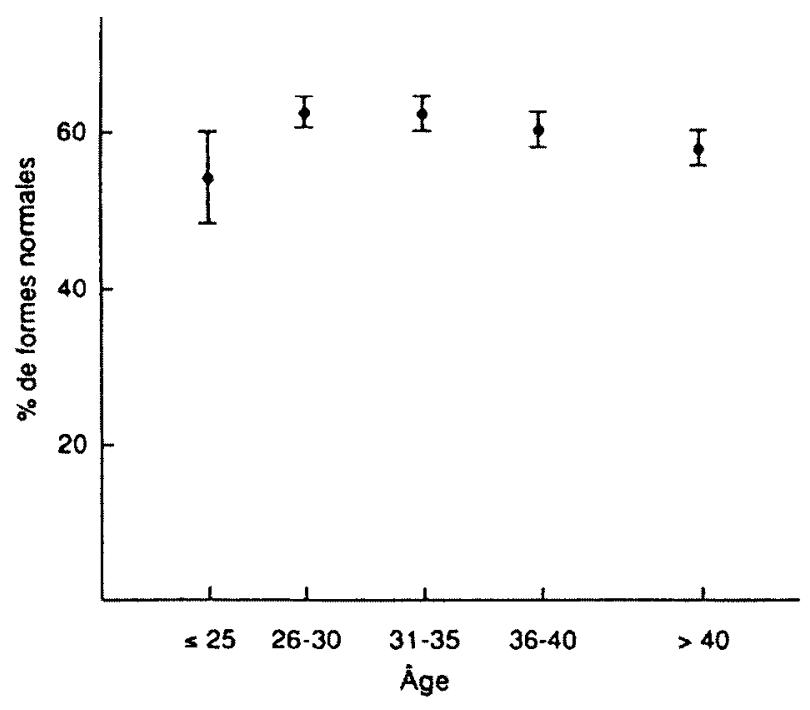

Figure 3 : Variation du pourcentage de formes normales des spermatozoüdes en fonction de l'âge du sujet chez l'homme [5].

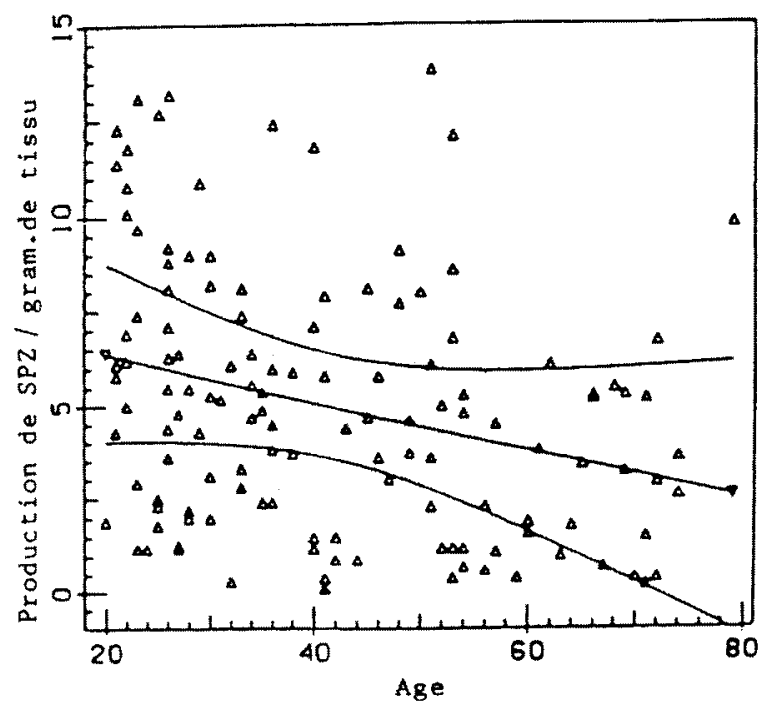

Figure 2 : Effet du vieillissement sur la production quotidienne de spermatozoüdes chez l'homme, par gramme de parenchyme. Les deux lignes courbes représentent la variation des écarts-types [4].

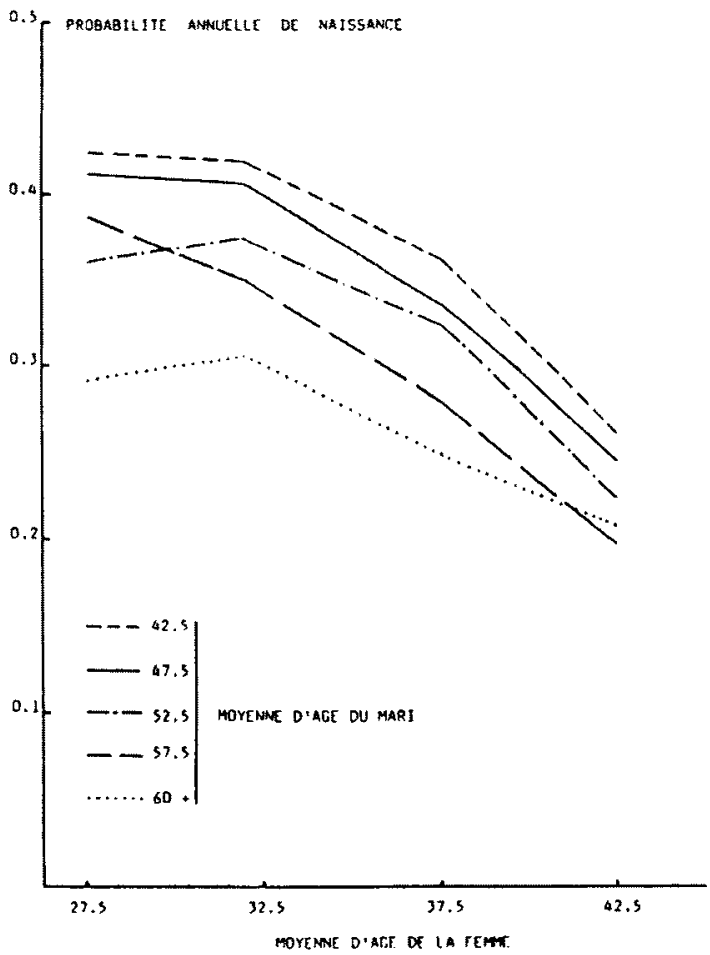

Figure 4 : Probabilité annuelle de naissance dans un couple, en fonction des âges respectifs du père et de la mère (population irlandaise de 1911, c'està-dire sans contraception organisée et avec un écart d'âge homme-femme fréquemment important) [9]. 
Tableau 1 : L'âge du père et la neurofibromatose de Recklinghausen [26].

Maladie de Recklinghausen

Population générale (INSEE)

Age paternel $\mathrm{m}=29,1 \pm 0,3$

Age maternel $\mathrm{m}=26,9 \pm 0,3$
Carey, 1979

32

27,5
Riccardi, 1984

32,8

27,4
Kaplan, 1987

34,9

27,8
Lesch-Nyhan ainsi que de certains désordres neurophysiologiques comme l'athétose, ou mentaux comme la schizophrénie. Si la fréquence de chacune de ces anomalies est très faible, leur nombre, environ 25 selon les estimations actuelles [revue : 25], multiplie évidemment les risques. Selon Friedman [26], la fréquence des anomalies dues au vieillissement paternel à partir de 40 ans atteindrait 0,3 à $0,5 \%$ des naissances, ce qui situerait le risque au niveau de la T21 pour une femme de 35-40 ans. Tandis que Hook critique ces estimations qui, selon lui, ne reflètent que les limites supérieures du phénomène [20] Lian et coll. [27], étudiant environ 7500 nouveau-nés anormaux, montrent que le risque d'ansmalie congénitale est de $2 \%$ lorsque le père à $20 \mathrm{ans}$, $2,4 \%$ lorsqu'il en a 40 et $2,6 \%$ lorsqu'il en a 50 . Cette augmentation de $0,6 \%$ se rapproche donc des résultats de Friedman.

D'autres anomalies présentent le caractère de mutations dominantes. Une première étude portant sur plus de 4000 cas de malformations cardiaques congénitales a montré que le nombre des anomalies des septa auriculaire et ventriculaire ou du canal artériel augmentait significativement à partir d'un âge paternel de 35 ans, les anomalies septales augmentant aussi lorsque cet âge décroît à partir de la vingtaine [28]. Une seconde étude, portant sur près de 10000 cas d'anomalies néonatales révèle, d'une part que le nombre d'altérations du tube neural, de cataractes congénitales et de T21 augmente régulièrement à partir d'un âge paternel de 30 ans et, d'autre part, que la fréquence des anomalies du tube neural, des hypospadias, des reins polykystiques et de la T21 augmente également quand l'âge du père décroît à partir de 20 ans [29]. Un des points les plus intéressants de ces deux études, que l'on retrouvera plus loin, est qu'une trop grande jeunesse paternelle paraît avoir les mêmes inconvénients que les débuts de la vieillesse.

Pour certains auteurs, des mutations récessives liées à l'X peuvent également résulter du vieillissement paternel, comme l'hémophilie A ou la myopathie de Duchenne. Dans ces cas, la première mutation apparaîtrait chez le grandpère maternel, serait transmise par ses filles et s'exprimerait chez la moitié de ses petits fils [30].

Des travaux récents ont enfin montré, à partir d'une étude des familles aristocratiques d'Europe dont la généalogie était connue et regroupant plus de 8500 adultes de 30 ans et plus, qu'un âge paternel de 50-59 ans (l'âge maternel étant constant) entraînait, chez les filles, une diminution de la longévité de 4,4 années relativement à celles nées de père de 20-29 ans. Les garçons n'étant pas touchés, les auteurs concluent que le chromosome $\mathrm{X}$ paternel ne se trouvant que chez les filles, celui-ci pourrait être porteur de gènes concernant la longévité et subissant des altérations dues au vieillissement [31].

\section{a) Origine des mutations}

Les mutations dont on vient de parler peuvent être dues à des facteurs exogènes (rayonnements, produits chimiques) ou à des facteurs endogènes. Ceux-ci ne sont généralement pas pris en compte et, pourtant, ils sont en rapport avec les caractères de la spermatogénèse. La probabilité d'apparition des anomalies géniques est en effet beaucoup plus grande chez le mâle que chez la femelle. On explique ce fait de la manière suivante. On sait que les phases de multiplications cellulaires sont sources de mutations géniques en particulier à cause d'erreur de recopiage du message génétique [30,32]. Chez la femelle, les ovogonies se sont multipliées pendant la phase fotale et c'est à partir de ce stock, sans multiplications nouvelles que, après la puberté, seront périodi- 
quement fournis les ovocytes. Chez le mâle au contraire, les spermatogonies, dont les multiplications avaient eu lieu pendant la phase fotale puis s'étaient arrêtées, vont se multiplier sans cesse à partir de la puberté et jusqu'à un âge très avancé (au delà de 80 ans), même si la production diminue graduellement. Pendant la période de pleine activité sexuelle, le rythme est d'environ 23 multiplications par an. Les spermatozoïdes d'un homme de 28 ans auront ainsi derrière eux, depuis la puberté, à peu près 380 divisions cellulaires, et ceux d'un homme de 35 ans à peu près 540 . On comprend mieux, à partir de ces données, l'augmentation du risque de mutation.

Une étude expérimentale vient enfin de montrer que les mutations spontanées de Lac I chez des souris transgéniques étaient:

- moins fréquentes dans les cellules germinales que dans les cellules somatiques,

- plus fréquentes dans les spermatogonies A que dans les cellules des stades ultérieurs,

- plus fréquentes dans les cellules germinales des souris vieillissantes, principalement de la méiose à la spermiogenèse. Cela peut suggérer une diminution, liée au vieillissement, de l'efficacité des contrôles méiotiques ou de celle des systèmes réparateurs [33].

Comme on vient de le voir, ces mutations peuvent être liées au grand nombre de multiplications cellulaires de la spermatogenèse. Mais elles peuvent aussi dépendre de l'accumulation des agents mutagènes de l'environnement et cela d'autant plus que :

a) l'activité des enzymes antioxydantes des spermatozoïdes et du plasma séminal qui limitent les dégradations dues aux radicaux libres diminuent avec l'âge [34] ;

b) les systèmes réparateurs de l'ADN disparaissent à partir du stade des spermatides âgées [35]. Il faut cependant signaler que l'ovocyte conserve lui-même, et pendant longtemps, un pouvoir réparateur efficace dont, dans une certaine mesure, pourront bénéficier le spermatozoïde et l'oeuf fécondé [36].

\section{Modifications fonctionnelles cérébrales}

Au delà des anomalies ponctuelles dont nous venons de parler, l'âge paternel paraît impliqué dans des variations subtiles et continues de la qualité du conceptus. Nous avons en effet montré que, à âge maternel constant, le vieillissement paternel entraînait :

- chez l'animal, une diminution progressive de la capacité d'apprentissage de la progéniture (Figure 5) [37].

- chez l'homme, une diminution de la réussite à des tests psychométriques [38]. Ces tests sont ceux qui sont effectués par les jeunes hommes du contingent, âgés de 18 ans, avant leur incorporation dans l'armée.

Une première étude à porté sur plus de 1700 recrues. L'influence du vieillissement paternel se manifeste à partir de la trentaine. Symétriquement, nous avons constaté qu'un très jeune âge paternel (post pubertaire) entraînait le même effet, les performances des enfants s'améliorant au fur et à mesure que l'âge du père augmentait, cela jusque vers 30 ans. La courbe générale de la réussite aux tests a donc la forme d'une parabole dont le sommet correspond à la trentaine paternelle (Figure 6). Lorsque c'est l'âge du père qui est maintenu constant, l'association entre la valeur de la note et l'âge de la mère n'existe pas : celui-ci n'aurait donc pas d'influence sur l'évolution des paramètres considérés.

Cette première enquête avait été faite à Nancy en 1985 et l'on pouvait imaginer, pour expliquer la courbe, l'existence d'un effet cohorte introduisant un biais. Nous avons donc refait le même travail dans une autre région, à une autre époque et sur un nombre plus important de cas :

- la région a été l'ensemble de Paris - Ile de France, où les brassages de population sont considérables ;

- l'enquête s'est étalée sur 1989 et 1990 et a concerné environ 11000 recrues.

L'analyse des 5000 premiers cas fournit le même type de courbe que précédemment (figure 7) [39]. Ces données dépassent évidemment le cadre de la pathologie et concernent les facteurs généraux pouvant influer sur la qualité du conceptus. Dans cette perspective, il faut faire la part des facteurs culturels et vérifier la 


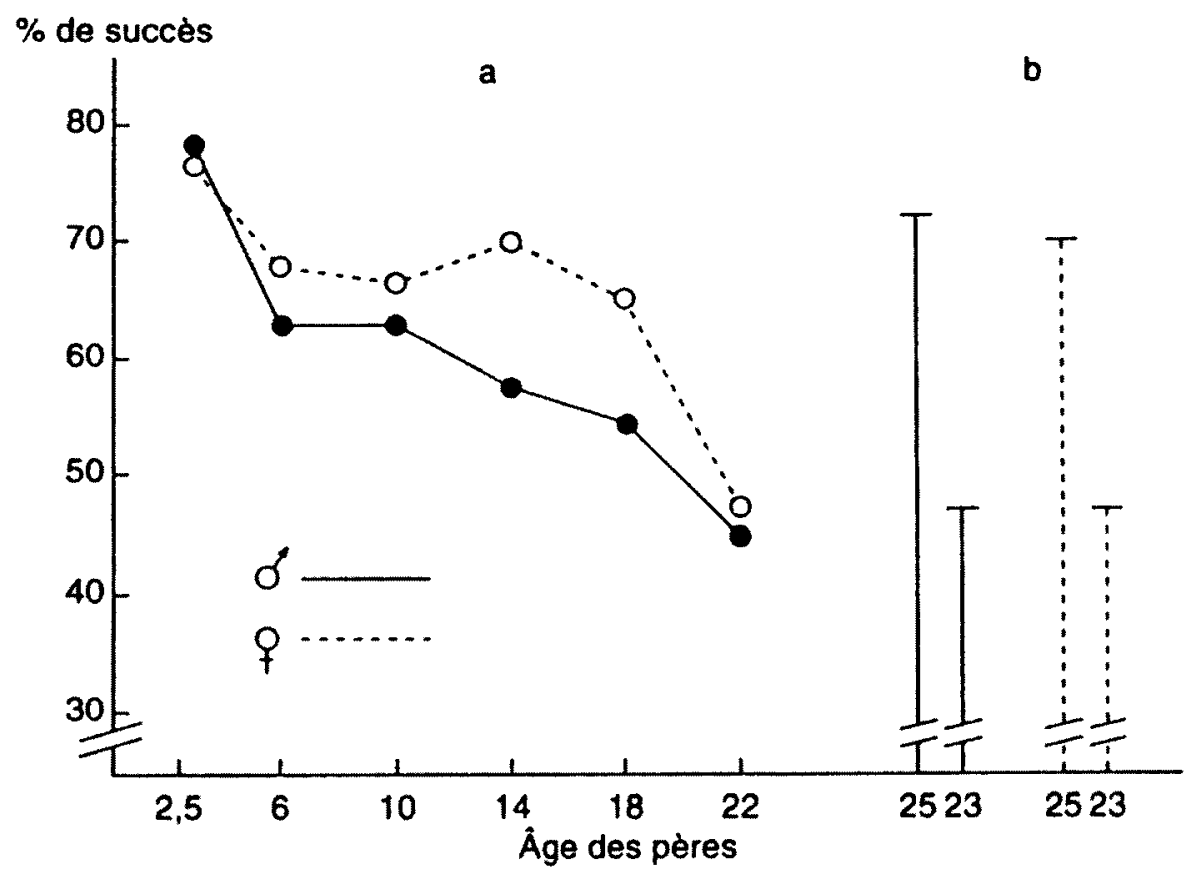

Figure 5 : a) Un même groupe de rats, successivement croisés à 2.5, 6, 10, 14, 18 et 22 mois avec des femelles de 2.5 mois, engendre des petits qui, adultes (2,5 mois) sont de moins en moins capables d'apprendre à éviter une stimulation nociceptive (courant électrique) succédant à une stimulation sensorielle banale (son + lumière) (Test du shuttle-box : chaque animal est soumis à une série de 50 essais, séparés chacun de 30 secondes ; lorsqu'il effectue 6 bons essais de suite, on considère que l'animal a réussi l'apprentissage). Les mâles sont d'emblée plus atteints que les femelles, celles-ci n'étant significativement concernées par le phénomène que lorsque le père est âgé de 22 mois (on peut considérer que cet âge correspond à 65.70 ans chez l'homme) ; il est actuellement impossible de fournir une explication génétique claire à cette différence sexuelle. Remarquons que la plus grande fragilité du mâle impliquée par ce résultat existe chez l'homme, dans la mesure où une population de débiles mentaux comporte significativement plus de garçons que de filles [52], ce qui pourrait être mis sur le compte du syndrome de l'X fragile.

b) Des rats de 2,5 et 23 mois, simultanément croisés avec des femelles de 2,5 mois, engendrent des petits dont les capacités d'apprentissage sont significativement plus faibles quand le père est âgé (expérience faite pour confirmer la précédente) [37].

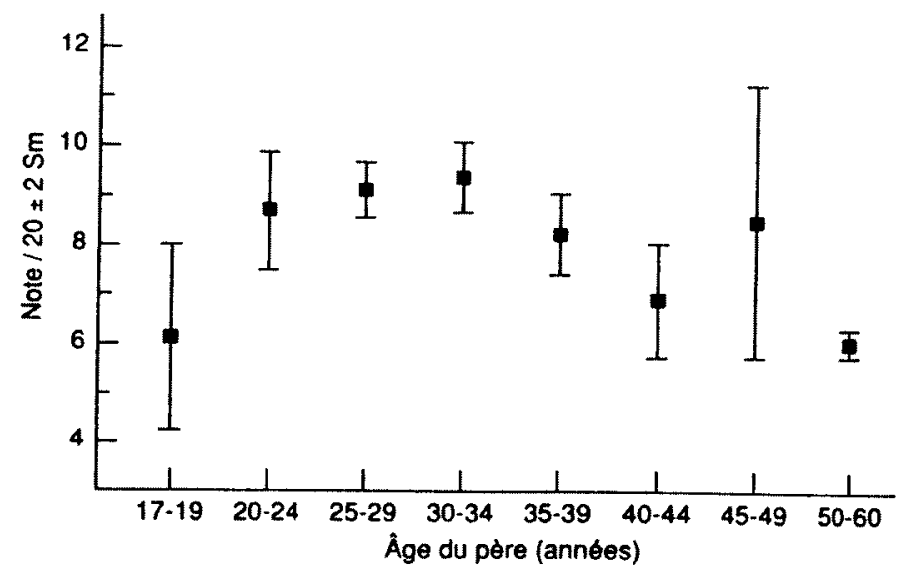

Figure 6 : Age paternel et qualité de la progéniture. L'âge du père à la naissance influe sur la probabilité de réussite de jeunes hommes de 18 ans $\grave{a}$ des tests psychométriques $(P<$ $0,04)$. La courbe présente une forme parabolique [38]. 


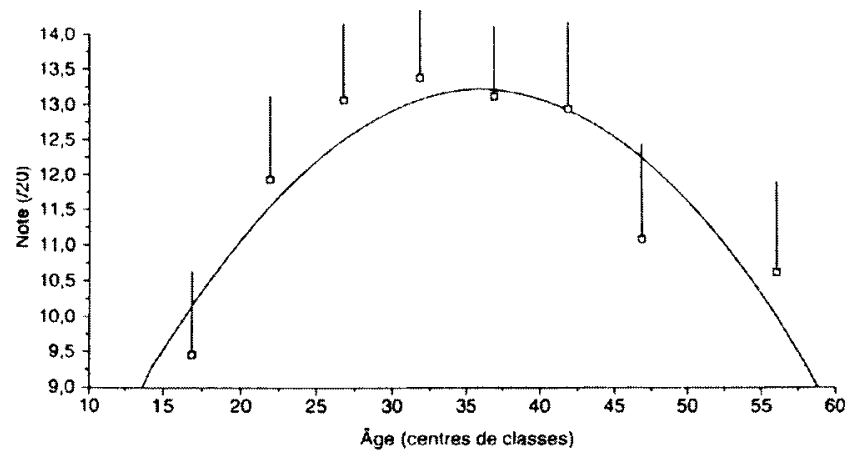

Figure 7 : Age paternel et qualité de la progéniture. Résultats partiels de l'enquête en cours. L'analyse des 5000 premiers cas aboutit à une courbe semblable à celle de la Figure 6 [39].

neutralité de l'âge maternel : c'est un des buts de notre deuxième enquête lorsque celle-ci aura pris en compte les 11000 cas prévus. $\mathrm{Si}$ toutefois on fait intervenir dès maintenant l'hypothèse génétique, la forme de la courbe signifie qu'une amélioration très subtile pourrait caractériser le génome du spermatozoïde à partir de la puberté, la qualité de celui-ci passant par un maximum autour de 30 ans, pour se dégrader ensuite. Or c'est, comme on l'a vu, ce que l'on constate pour les caractères qualitatifs des spermatozoides qui passent par un maximum, chez l'homme, aux alentours de la trentaine [5]. Puisqu'on sait que la morphologie du spermatozoïde est génétiquement déterminée [40], une telle évolution suggère une amélioration des facteurs informatifs dans la partie ascendante de la courbe, et une dégradation de ces facteurs dans la partie descendante. On peut donc se demander si des phénomènes analogues ne pourraient pas concerner d'autres informations que celles qui déterminent le phénotype des gamètes, c'est à dire celles qui déterminent le phénotype de l'indivi$\mathrm{du}$.

La dégradation des facteurs informatifs dans la partie descendante est facilement expliquée par les phénomènes du vieillissement qui, comme ils touchent les cellules somatiques, touchent sans doute les spermatogonies puisque des systèmes réparateurs d'ADN y ont été mis en évidence [41]. Des travaux ont par ailleurs montré que l'ADN mitochondrial était plus fragile que celui du noyau vis-à-vis des mutations, en particulier vis-à-vis de celles dues aux radicaux libres, qui s'accumulent avec l'âge [42]. Ces altérations entraînant une chute du potentiel des phosphorylations oxydatives des mitochondries on a là, bien que les mitochondries du spermatozoïde ne représentent que $0,1 \%$ du stock mitochondrial du zygote, un autre impact possible du vieillissement masculin.

Quant à la nature et à la subtilité des troubles observés, il faut les rapprocher des résultats que nous avons déjà obtenus en provoquant chez le rat mâle des mutations expérimentales à l'aide d'antimitotiques mutagènes : la progéniture de ces mâles présente en effet une altération de la capacité d'apprendre [43], soustendue par une chute de certains des supports biochimiques de la mémoire [44].

Il est plus difficile d'expliquer l'amélioration des facteurs informatifs dans la partie montante de la courbe. Cependant, le rôle actuellement attribué au cytoplasme dans l'arrangement final de l'ADN des gamètes $[45,46,47$, 48] permet d'envisager l'existence d'une période de maturation qui, pendant un temps plus ou moins long, pourrait précéder la maturité du génome. Dans ce contexte, nous avons récemment exploré le phénomène chez la souris. L'expérience a montré, d'une part que l'activité spontanée et les comportements d'exploration (figure 8), d'autre part les capacités d'apprentissage (figure 9), étaient moins développés chez des animaux nés de pères immédiatement post-pubères $(6,5$ sem) que chez ceux nés de pères adultes (12 et $16 \mathrm{sem}$ ) [49]. Quant à l'apparente neutralité maternelle elle pourrait, si elle se confirme, être expliquée par les rôles différents et complémentaires que jouent les génomes maternel et paternel dans le développement du conceptus $[50,51]$. Si les informations fournies par le père et la mère ne sont pas équivalentes, on peut en effet admettre que des changements dans l'un ou l'autre génome ne produiront pas les mêmes effets et que, en l'occurrence, les informations paternelles jouent un rôle prédominant dans les caractères que nous avons explorés. 

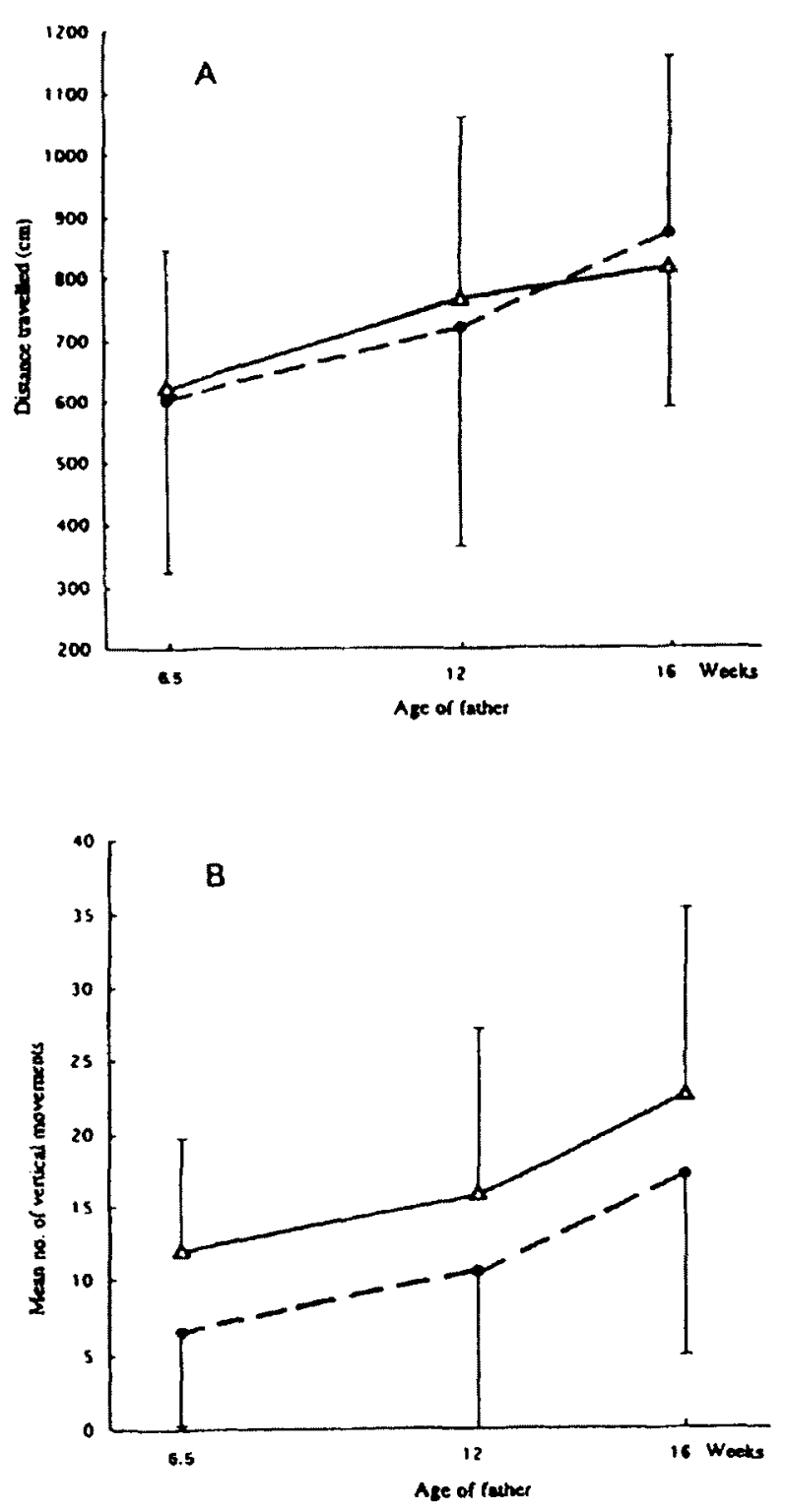

Figure 8: Mesure de l'activité spontanée moyenne chez des souris nées de pères post-pubères (6,5 sem) et adultes (12 et 16 semaines)(ces animaux résul. tent du croisement de mâles consanguins CBA/JICo et de femelles consanguines C57BL/6.JICo). Test du champ libre, durée : 3 min. A : distance parcourue $\Delta$ : mâles, $r P<0.001 ; O$ : femelles, $r: P<0.01 ; B$ : mouvements verticaux (comportements exploratoires) $\Delta$ et $O: r: P<0.001$ [49].
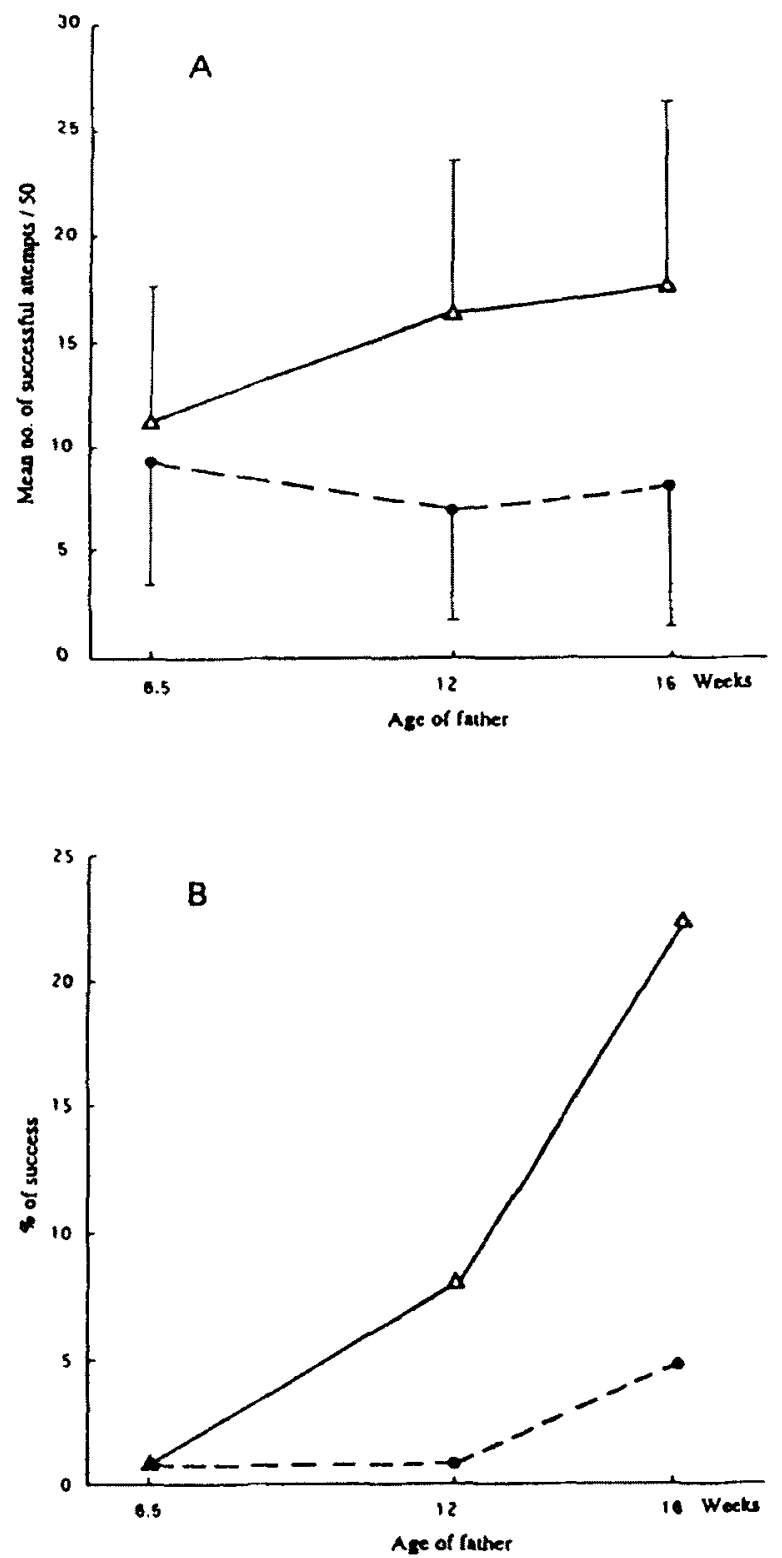

Figure 9 : Capacités d'apprentissage des mêmes souris que celles concernées par la figure 8 . Même test que celui de la figure $5 . A$ : nombre moyen de bons essais $/ 50$. B : pourcentage d'animaux ayant réussi l'apprentissage (6 bons essais de suite). Les performances n'augmentent significativement que chez les mâles $(r: P<0.001)$ [49]. 


\section{CONCLUSION}

Ainsi l'âge paternel, qui intervient dans l'apparition de syndromes parfaitement définis, interviendrait aussi dans la détermination des plus fines potentialités du conceptus. S'il se confirmait, ce dernier aspect du problème serait particulièrement important puisqu'il concernerait non plus l'apparition ponctuelle d'une symptomatologie, mais un continuum de qualité intéressant la population en général. Que les facteurs en cause soient psychosociaux, génétiques ou les deux à la fois, il serait d'autant plus utile de les répertorier qu'indépendamment du comportement ils pourraient surtout si le génome est en cause, agir sur d'autres paramètres de la vie comme les moyens de défenses de l'organisme, la morbidité, le vieillissement de l'individu, etc., et l'on a vu, par exemple, que la longévité des filles pouvait être concernée. Le recensement de ces facteurs serait alors aussi utile que celui des toxiques de l'environnement afin d'essayer d'obtenir, cette fois dans le cadre de la normalité et pour un patrimoine génétique donné, des conceptus de qualité maximum.

\section{REFERENCES}

1 TAKIZAWA T., HATAKEYAMA S. - Age associated changes in microvasculature of human adult testis. Acta Path. Jap., 1978, 28, 541-554.

2 AUROUX M. - Le vieillissement testiculaire et son influence sur la progéniture. In Andrologie, G. Arvis ed. Maloine, Paris, 1989, 753-761.

3 ROBIN C. - Le sperme. In Dictionnaire encyclopédique des sciences médicales. A. Dechambre ed., Masson et Asselin, Paris, 1883, 3e série, XI, 112-193.

4 JOHNSON L. - Spermatogonia and Aging in the human. J. Androl., 1986, 7, 331-354.

5 SCHWARTZ D., MAYAUX M.J., SPIRA A., MOSCATO M.L., JOUANNET P., CZYGLIK F., DAVID G. Study of a group of $\mathbf{4 8 4}$ fertile men, Part II : Relation between age (20-59) and semen charcteristics. Int. J. of Androl., 1981, 4, 450-456.

6 ALBERT M., ROUSSEL C. - Strain differences in the concentration, mobility and morphology of epididymal sperm in relation to puberty in mice. Int. J. Androl., 1984, 7, 334-347.

7 LUNSTRA D.D., ECHTERNKAMP S.E.- Puberty in beef bulls : acrosome morphology and semen quality in bulls of different breeds. J. Anim. Sci., 1982, 55, 638-648.
8 MacLEOD J., GOLD. R. Z. -The male factor in fertility and infertility. VI : Semen quality and certain others factors in relation to ease conception. Fertil. Steril., 1953, 4, 10-33.

9 ANDERSON B.A. - Male age and infertility. Result from Ireland prior to 1911. Pop Index., 1975, 41, 561567.

10 Fédération CECOS. Female fecundity as a function of age. N. Engl. J. Med., 1982, 306, 404-406.

11 MARTIN R.H, RADEMAKER A.W.- The effect of age on the frequency of sperm chromosomal abnormalities in normal men. Am. J. Hum. Genet. 1987, 41, 484-492.

12 MARTIN R.H., BALKAN W., BURNS K., RADEMAKER A.W., LIN C.C., RUDD N.L. The chromosome constitution of 1000 human spermatozoa. Hum. Genet. 1983, 63, 305-309.

13 BRANDRIFF B., GORDON L., ASHWORTH L., WATCHMAKER G., MOORE II D., WYROBEK A.J., CARRANO A.V. - Chromosomes of human sperm : variability among normal individuals. Hum. Genet. $1985,70,18-24$

14 SHERMAN S.L., TAKAESU N., FREEMAN S; PHILLIPS C., BLACKSTON R.D., KEATS B.J., JACOBS P.A., COCKWELL A.E., KURNITE D., UCHIDA I., HASSOLD T.J. - Trisomy 21 : association between reduced recombination and non disjunction. Am. J. Hum. Genet. 1990, 47, A 97.

15 STYLIANOS E., ANTONARAKIS M.D. - The down syndrom collaborative group. Parental origin of the extra chromosome in trisomy 21 as indicated by analysis of DNA polymorphisms. N. Engl. J. Med. 1991, $324,872-876$.

16 STENE J., FISCHER G., STENE E., MIKKELSEN M; PETERSEN E. - Paternal age effect in Down's syndrome. Ann Hum. Genet. 1977, 40, 299-306.

17 MATSUNAGA E., TONOMURA E., OISHI A., KIKUCHI Y. Reexamination of paternal age effect in Down's syndrome. Hum. Genet. 1978, 40, 259-268.

18 CECOS, THEPOT F., WACK T., SELVA J., CZYGLIK F., MAYAUX M.J. - Age paternel et issues de grossesses. Expérience des CECOS. Contracept. Fertil. Sex. 1993, 21, 388-390.

19 ROTH M.P., STOLL C., TAILLEMITE J.L., GIRARD S., BOUE A. - Paternal age and Down's syndrome diagnosed prenatally : no association in French data. Prenat. Diagn. 1983, 3, 327-335.

20 HOOK E.B., REGAL R.R. - A search of a paternalage effect upon cases of $47,+21$ in wich the extra chromosome is of paternal origin. Am. J. Hum. Genet. 1984, 36, 413-421.

21 HASSOLD T.J. - The origin of non-disjunction in humans. Meeting of the International Human Genetics Society. Washington, October 1991. In Ségolène AYME : Epidémiologie de la Trisomie 21. Les données récentes. 10ème séminaire de 
Diagnostic Anténatal des malformations. Paris (Necker) 1991.

22 HOOK E.B., SCHREINEMACHERS D.M., WILLEY A.M., CROSS P.K. Inherited structural cytogenetic abnormalities detected incidentally in fetuses diagnosed prenatally : frequency, paternal age associations, sex-ratio trends, and comparisons with rates of mutants. Am. J. Hum. Genet. 1984, 36, 422-443.

23 PENROSE L.S. - Paternal age and mutation. Lancet, 1995, II, 312-313.

24 KAPLAN J., TOUTAIN A. - La maiadie de Recklinghausen. In 3e Sem. Genet. Clin. : affections dominantes à expression variable et Conseil Génétique, Paris, Necker, 1987, 42-52.

25 TARIN J.J., BRINES J., CANO A. -Long term effects of delayed parenthood. Hum. Reprod., 1998, 13 : 2371-2376

26 FRIEDMAN J.M. - Genetic desease in the offspring of older fathers. Obstet. Gynecol., 1981, 57, 745-749.

27 LIAN Z.H., ZACK MM., ERICKSON J.D. Paternal age and the occurence of birth defects. Am. J. Hum. Genet. 1986, 39, 648-660.

28 OLSHAN A.F., SCHNITZER P.G., BAIRD P.A. Paternal age and the risk of congenital defects. Teratology, 1994, $50: 80-84$.

29 Mc INTOSH G.C., OLSHAN A.F., BAIRD P.A. Paternal age and the risk of birth defects in offspring. Epidemiology, 1995, $6: 282-288$.

30 VOGEL F., RATHENBERG R. - Spontaneous mutation in man. Adv. Hum. Genet., 1975, 5, 223-318.

31 GAVRILOV L.A., GAVRILOVA N.S., KROUTKO V.N., EVDOKUSHKINA G.N., SEMYONOVA V.G., GAVRILOVA A.L., LAPSHIN E.V., EVDOKUSHKINA N.N., KUSHNAREVA Y.E. - Mutation load and human longevity. Mutat. Res., 1997, 377 : 61-62.

32 CROW J.F. - The high spontaneous mutation rate : is it a health risk? Proc. Natl. Acad. Sci. USA, 1997, 94: 8380-8386.

33 WALTER C.A., INTANO G.W., McCARREY J.R., McMAHAN C.A., WALTER R.B. - Mutation frequency declines during spermatogenesis in young mice but increases in old mice. Proc. Natl. Acad. Sci. USA, 1998, $95: 10015-10019$.

34 KELSO K.A., REDPATH A., NOBLE R.C. SPEAK B.K. - Lipid and antioxidant changes in spermatozoa and seminal plasma throughout the reproductive period of bulls. J. Reprod. Fertil., 1997 : 109 : 1-6.

35 INOUE M., KURIHARA T., YAMASHITA M., TATSUNI K. Effects of treatment with methyl methane sulfonate during meiotic and post meiotic stages and maturation of spermatozoa in mice. Mutat. Res., 1993, $294: 179-186$.

36 ASHWOOD-SMITH M.J., EDWARDS R.G. - DNA repair by ovocytes. Mol. Hum. Reprod., 1996, 2 : 4651.
37 AUROUX M. - Decrease of learning capacity in offspring with increasing paternal age in the rat. Teratology, 1983, 27, 141-148.

38 AUROUX M., MAYAUX M.J., GUIHARD-MOSCATO M.L., FROMANTIN M., BARTHE J., SCHWARTZ D. - Paternal age and mental functions of progeny in man. Hum. Reprod., 1989, 4, 794-797.

39 AUROUX M. - Paternal age and development. In : Fertility and Sterility. A current overview. B. Hedon, J. Bringer and P. Mares eds. Proc. 15 th World Congress on Fertility and Sterility, Montpellier, France, 17-22 sept. 1995, Parthenon Publishing Group (London), p 321-328.

40 BURGOYNE P.S. - Sperm phenotype and its relationship to somatic and germ line genotype : a study using mouse agregation chimeras. Dev. Biol., 1975, 44, 63-76.

41 LEE I.P. - Adaptative biochemical repair responses toward germ cell DNA damage. Am. J. Int. Med., 1983, 4, 135-147.

42 WALLACE D.C. - Mitochondrial genetics : a paradigm for aging and degenerative diseases? Sciences, $1992,256,628-632$.

43 AUROUX M., DULIOUST E., SELVA J., RINCE P Cyclophosphamide in the F0 male rat : physical and behavioral changes in three successive adult generations. Mutat. Res., 1990, 229, 189-200.

44 AUROUX M.R, DULIOUST E.M., NAWAR N.Y, YACOUB S.G., KEMPF E.H, EBEL A.B.Cyclophosphamide in the male rat : cerebral biochemical changes in progeny. Biomed. Pharmacother., 1990, 44, 519-523.

45 CAMPBELL J.H., ZIMMERMANN E.G. Automodulation of genes : a proposed mechanism for persisting effects of drugs and hormones in mammals. Neurobehav. Toxicol. Teratol., 1982, 4, 435-439.

46 CAMPBELL J.H. - Automodulation of genes : explanation for lasting effects seen in fuctionnal neuroteratology ? Presented at the 15th Intern. Summer School of Brain Research : Neurochemistry of Functionnal Neuroteratology, Amsterdam, August 31 to september 4, 1987, 33.

47 REIK W., WALTER J., GURTMANN I., FEIL R., SURANI A., SASKI H., KLOSE J., ALLEN N. Imprinting in development and disease. 9 th Annual meeting of European Society of Human Reproduction and Embryology, THESSALONIKI 27-30 June 1993.

48 KRUMLAUF R., MARSHALL H., MONCHEV S., SHAM M.H., STUDER M. - Transgenic approaches to the role of Hox homeobox genes in craniofacial development. 21 st Annual conference of the European Teratology Society. Lyon 6-9 Septembre 1993.

49 AUROUX M., NAWAR N.N.Y., NAGUIB M., BAUD M., LAPAQUELLERIE N. - Post-pubescent to mature fathers : increase in progeny quality? Hum. Reprod., 1998, $13: 55-59$. 
50 BARTON S.C, SURANI M.A.H., NORRIS M.L. - Role of paternal and maternal genome in mouse development. Nature, 1984, 311, 374-376.

51 SAPIENZA C., PETERSON A.C., ROSSANT J., BALLING R. - Degree of methylation of transgenes is dependent on gamete of origin. Nature, 1987, 328, 251-254.

52 ANTHENAISE M., SALBREUX R. - Prévalence de la déficience mentale profonde chez l'enfant. Neuropsy, Enfance Adolesc., 1979, 27, 45-48.

\section{ABSTRACT \\ Paternal age and progeny \\ M. AUROUX}

Testicular ageing affects at the same time the individual and his lineage. In the individual, vascular, endocrine, blood testis barrier and Sertoli cells changes because of age lead a decrease of spermatozoa number and an alteration in their form and motility. These changes lead a gradual decrease of fertility. In the progeny, paternel ageing increases the risk of new dominant autosomic mutations which themselves cause different malformations or functional disorders and that of certain chromosome $X$ linked recessive mutations. Moreover, it seems responsible for a decrease of daughters'longevity. Finally, in animal an man, very youthful age and paternal are accompanied by a lowering in the level of progeny cognitive functions. Maternal age did not appear to play a part in this event. On the whole, these results pose the problem of the optimum age for fatherhood.

Key words : Paternel age, spermatozoa, fertility, mutations, progeny. 